\title{
Comparative Study of Bend Loss in Large Mode Holey and Conventional Fibres
}

\author{
Tanya M. Monro, Joanne C. Baggett Kentaro Furusawa and D. J. Richardson. \\ Optoelectronics Research Centre, University of Southampton, UK
}

\begin{abstract}
We present the first comparison of bend losses in large mode holey and conventional fibres, demonstrating holey fibres to be a practical alternative, with the additional benefit of broadband singlemode guidance and tailorable bend loss.
\end{abstract}

Single mode fibers with large mode areas are necessary for applications requiring high power delivery including $\mathrm{Er}$ and $\mathrm{Yb}$ fiber lasers and amplifiers. Conventional approaches to increasing mode area rely on reducing the numerical aperture, which has accuracy limitations, although often the real practical limitation is due to bend loss. Recently, holey fiber technology has been proposed as an alternative route towards large mode areas [1]. In a holey fiber (HF), light is guided by air holes which form the cladding. The optical properties are strongly wavelength dependent and thus sensitive to the separation $\Lambda$ and diameter $d$ of the holes, which can be specified during fabrication. One striking property of HFs is that they display endlessly single moded guidance when $d / \Lambda$ is small [2]. Despite this advantage, little quantitative information exists regarding the bend-loss and mode area characteristics of holey fibers.

We present a comparative study of mode area $\left(\mathrm{A}_{\mathrm{eff}}\right)$ and bend loss for a range of large mode holey and conventional fibers [3]. Here a selection of results from a study of HFs for which $7.5 \leq \Lambda \leq 15 \mu \mathrm{m}$ and 0.6 $\leq \mathrm{d} \leq 2.4 \mu \mathrm{m}$ are presented (labelled H1-6). Conventional telecommunications and large mode area (LMA) fibers are included for comparison (S1-2), where the LMA fibre considered here has NA $\approx 0.06$ and core diameter $\approx 18 \mu \mathrm{m}[4]$. The mode area of each fiber is extracted from divergence measurements and shown in Fig. 1. Fibre $\mathrm{H} 1$ is single moded at all wavelengths and has $A_{\text {eff }}=680 \mu \mathrm{m}^{2}$ at $1.55 \mu \mathrm{m}$, substantially larger than fiber S1, $\left(\mathrm{A}_{\text {eff }}=405 \mu \mathrm{m}^{2}\right)$, which is typical of the largest fundamental modes achieved in conventional fibers. The $\mathrm{A}_{\text {eff }}$ of fiber $\mathrm{H} 2$ is identical to $\mathrm{S} 2$ at $1.55 \mu \mathrm{m}$ but varies considerably less with wavelength, which is typical of holey fibers.

Figure 2 shows numerical predictions for how $A_{\text {eff }}$ varies with $\mathrm{d} / \Lambda$ and $\Lambda$ at $1.53 \mu \mathrm{m}$ (calculated using the model in Ref. [5]). Note that the same $A_{\text {eff }}$ can result from a range of different structures, and that $A_{\text {eff }}$ is more sensitive to $d / \Lambda$ than $\Lambda$ when $d / \Lambda$ is small.

We have measured the bend loss at $1.5 \mu \mathrm{m}$ in large mode conventional and holey fibers, and a selection of these results are shown in Fig. 3. Although a bigger mode gives rise to higher bend loss in conventional fibers, our results indicate that mode area is not the sole determinant of bend loss in a holey fiber. Notice that H4 has a larger mode than $\mathrm{H} 3$ but lower bend loss, and that $\mathrm{H} 2$ has the same $\mathrm{A}_{\text {eff }}$ as standard telecommunications fiber with reduced bend loss. These results indicate that bend loss can be optimized by careful choice of structure $(\mathrm{d} / \Lambda, \Lambda)$, and that bend losses can be reduced using structures with larger holes (however for $\mathrm{d} / \Lambda \gtrsim 0.2$ the range of single-mode operation is reduced).

In conclusion, our comparative study of large mode holey and conventional fibers demonstrates that holey fibers are a versatile alternative to large mode conventional fibers, particularly for broadband applications where the added benefit of endless single mode guidance is valuable. Bend losses in these fibers are comparable with conventional fibers, with the added advantage that the bend loss can be modified via the holey fiber geometry. 




Fig. 1. Mode area for a range of large mode fibers. H1-6 corresponds to holey fibers, S1 and S2 corresponds to conventional fibers [4]. Fibre dimensions are indicated in fig 2.



Fig. 2. Numerical predictions of $A_{\text {eff }}$ as a function of the structural parameters $d / \Lambda$ and $\Lambda$. Structures corresponding to holey fibers are indicated by H1-6. Contours show constant $A_{\text {eff }}$ in $\mu \mathrm{m}^{2}$. 


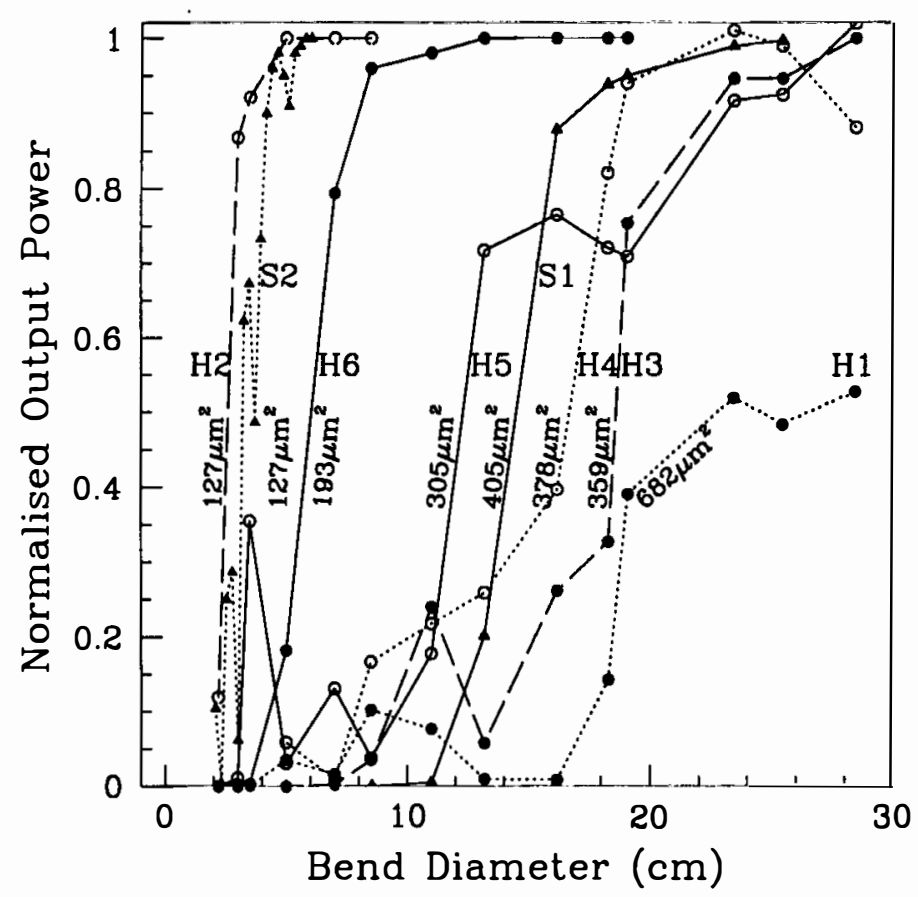

Fig. 3. Bend loss characteristics for a selection of large mode fibers studied.

1. J.C. Knight, T.A. Birks, R.F. Cregan, P.St.J. Russell and J.P. de Sandro, 'Large mode are photonic crystal fibre', Elect. Lett. 34 1346-1347 (1998).

2. T.A. Birks, J.C. Knight, and P.St.J. Russell "Endlessly single-mode photonic crystal fiber," Opt. Lett. 22 961-963 (1997).

3. G. Agrawal, Nonlinear Fiber Optics, Academic Press (1995), Sections 1.2.3 and 2.3.1.

4. N.G.R. Broderick, H.L. Offerhaus, D.J. Richardson, R.A. Sammut, J. Caplen and L. Dong, 'Large mode area fibers for high power applications', J.Opt. Fiber Tech. 5 185-197 (1999).

5. T.M. Monro, D.J. Richardson, N.G.R. Broderick and P.J. Bennett, 'Holey optical fibers: an efficient modal model', J. Lightw. Tech. 17 1093-1102 (1999). 